\title{
Civilisations
}

Revue internationale d'anthropologie et de sciences

humaines

58-2 | 2009

Intimités et inimitiés du religieux et du politique en

Afrique

\section{Constructions religieuses du politique}

Aux confins de Bamako (Mali)

\section{Françoise Bourdarias}

\section{(2) OpenEdition}

\section{Journals}

Édition électronique

URL : http://journals.openedition.org/civilisations/2070

DOI : $10.4000 /$ civilisations. 2070

ISSN : 2032-0442

Éditeur

Institut de sociologie de l'Université Libre de Bruxelles

Édition imprimée

Date de publication : 30 décembre 2009

Pagination : 21-40

ISBN : 2-87263-027-9

ISSN : 0009-8140

Référence électronique

Françoise Bourdarias, «Constructions religieuses du politique », Civilisations [En ligne], 58-2 | 2009, mis en ligne le 30 décembre 2012, consulté le 20 avril 2019. URL : http://journals.openedition.org/ civilisations/2070 ; DOI : 10.4000/civilisations.2070 


\title{
Constructions religieuses du politique \\ aux confins de Bamako (Mali)
}

\author{
Françoise BOURDARIAS
}

Résumé : Cet article présente quelques éléments de réflexion sur les formes d'articulation du religieux et du politique. Ils sont issus de recherches menées depuis 1993 au Mali, dans le quartier de Bankoni, situé à la périphérie de Bamako. Le développement de différents mouvements religieux dans cette zone urbaine a été observé entre 2000 et 2005, au moment où l'affiliation à un collectif religieux était présentée par les individus eux-mêmes comme un remède au "désordre 》 et à la transformation des liens sociaux. En fait, la configuration religieuse locale se caractérise par l'interdépendance de mouvements concurrents, musulmans ou issus des " religions traditionnelles 》, dont les dirigeants proposent une interprétation des perturbations sociales et économiques vécues par des populations particulièrement précarisées, et une remise en ordre de l'espace de vie et des relations sociales. L'analyse privilégie les constructions pratiques et symboliques élaborées par les fidèles, qui sélectionnent et réinvestissent des éléments des traditions religieuses proposées.

Mots-clés : politique, Islam, religions traditionnelles, Mali, urbanisation, mobilisations sociales.

\begin{abstract}
This article presents some thoughts on the relations between religion and politics. These thoughts have emerged from fieldwork conducted since 1993 in Mali, in the neighbourhood of Bankoni, in the suburbs of Bamako. The development of different religious movements in this urban area was observed between 2000 and 2005, in days when the affiliation to a religious association was presented by individuals themselves as a solution to "disorder " and to the transformation of social relationships. Actually, the local religious configuration is nowadays characterized by the interdependency of competing religious movements, Islamic and coming from a " traditional religion " background, whose elites offer both an interpretation of the social and economic turmoil experienced by very impoverished populations, and a new ordering of the space of everyday life and of social relationships. The analysis highlights the practical and symbolic constructions elaborated by the faithful of the diverse religious movements, who select and reinvest elements of the religious traditions that they have embraced.
\end{abstract}

Keywords: Politics, Islam, traditional religions, Mali, urbanization, social mobilizations. 


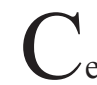

et article $^{1}$ propose quelques éléments de réflexion sur les formes d'articulation du religieux et du politique. Ils sont issus d'observations menées au sein d'une configuration sociale restreinte, singulière, bien que les dynamiques qui s'y inscrivent entretiennent de multiples affinités avec celles qui ont pu être décelées à la périphérie de nombreuses villes d'Afrique de l'Ouest.

$\mathrm{Au}$ nord de Bamako, Bankoni est une zone récemment urbanisée en extension « spontanée » régulière ${ }^{2}$, encore peuplée pour l'essentiel de foyers en situation d'insécurité économique, travailleurs précaires, chômeurs, petits artisans et commerçants. Les procédures de lotissement engagées depuis 1992 par les autorités du gouvernorat, la dévaluation du FCFA, la fermeture des dernières entreprises d'État, ont provoqué de profondes perturbations (Bourdarias 1999, 2007). Selon les habitants, « le désordre s'est installé », les règles qui régissaient les relations sociales ont été bouleversées.

D’année en année, depuis $1993^{3}$, j'ai pu appréhender la variété des stratégies individuelles et collectives élaborées dans ce contexte, de la révolte (luttes foncières) au refus explicite de « la politique », en passant par les tentatives d'insertion individuelle dans les réseaux de dépendance et de clientélisme liés à l'État ou aux ONG (Bourdarias 2003).

À la fin des années 1990, le développement des mouvements religieux dans l'ensemble des quartiers périurbains bamakois (mouvements islamiques réformistes ou fondamentalistes, communautés soufies, " néo fétichisme » urbain) semblait révéler de nouvelles formes de mobilisation de la jeunesse urbaine.

L'un des mouvements dont il sera question ici, l'Ançar-Dine, a installé son siège à Bankoni en 1991 (après la chute de Moussa Traoré). En quelques années, cette association islamique réformiste s'est implantée dans toute l'Afrique de l'Ouest, au Congo, dans plusieurs pays européens et aux États-Unis. Par ailleurs, les jeunes soufis sont de plus en plus nombreux à parcourir la zone, certains y fondent des établissements secondaires, manifestant ainsi le rayonnement de leur maître (karamoko ${ }^{4}$ ). Soufi Bilal et Soufi Adama sont considérés à Bankoni comme les maîtres les plus prestigieux. Dans la même période, les «féticheurs »

1. Ce texte est issu d'une communication présentée au colloque "Une opposition qui ne dit pas son nom ». Représentations et pratiques populaires (autour du pouvoir) au Burkina Faso et dans la sous-région. Université catholique de Louvain, 28 et 29 septembre 2006.

2. Bankoni (Commune I de Bamako) a vu le nombre de ses habitants à peu près doubler depuis le recensement de 1997 (autour de 150.000 selon les dernières estimations issues du recensement de 2009). Les quartiers qui composent cette zone se sont multipliés et s'étendent aujourd'hui jusqu'aux limites du district urbain. Au-delà, les terres des villages ruraux sont progressivement urbanisées.

3. Résidant chaque année de trois à quatre mois par an dans cette zone, je me suis interrogée sur les formes d'inscription locales de tensions économiques et sociales dont la prise en compte m'a conduit à élargir de plus en plus l'espace d'observation et d'analyse : évolution des rapports entre les générations, entre hommes et femmes, dans un contexte de transformation des conditions de l'activité économique ; luttes foncières suscitées par les politiques publiques de « lotissement » des quartiers ; transformation des pratiques politiques et des productions imaginaires qui leurs sont liées.

4. Le terme karamoko désigne le maître spirituel, celui qui dispense son savoir, se distingue par sa sagesse et son pouvoir d'attraction. 
(bolitigi $i^{5}$ ) et les groupes de chasseurs se sont multipliés, proclamant leur fidélité à la « vraie tradition bamanan d'avant l'Islam ». Daouda Yattara est alors le bolitigi le plus présent dans les média et le plus controversé au Mali, ses adeptes et ses élèves sont nombreux dans le quartier.

Si cette nébuleuse religieuse est traversée de conflits plus ou moins ouverts, les pratiques des dirigeants et de leurs fidèles montrent l'étroite interdépendance des mouvements qui la composent. Les prêcheurs musulmans s'interpellent et s'affrontent dans les journaux, sur les ondes des radios indépendantes, à travers les enregistrements sur cassettes et DVD diffusés sur tous les marchés (Schulz 2006). Certains bolitigi utilisent également ces supports.

Cette effervescence est perçue par les habitants des quartiers périphériques où elle donne lieu à d'inlassables débats au sein des groupes d'amis et des foyers, à l'extérieur aussi, au centre-ville où elle ne manque pas d'inquiéter une partie des élites politiques et économiques.

Le phénomène sera donc ici saisi au moment où il devient visible, où les agents sociaux, affiliés ou non, tentent collectivement de lui donner du sens, où les transformations relationnelles qui l'accompagnent posent problème.

La situation religieuse locale dont je sélectionnerai ici quelques traits a été observée entre 2003 et 2006. L'échelle d'observation microsociale adoptée faisait apparaître la multiplicité des stratégies sociales élaborées par des groupes confrontés au chômage, au délitement des formes de protection sociale liées aux solidarités familiales. Dans un tel contexte, l'affiliation à un mouvement religieux était fréquemment présentée par les individus euxmêmes comme un remède au " désordre » et à la transformation des liens familiaux et conjugaux, parfois comme un " retour » à la " tradition du village ». L'observation des relations sociales qui s'élaboraient au sein des collectifs religieux, des pratiques de mise en ordre de l'espace urbain, de la vie quotidienne des fidèles, incitait à interroger à ce niveau les formes d'articulation du religieux et du politique.

\section{Le religieux et le politique : les remèdes aux désordres urbains}

L'analyse des formes d'articulation entre le religieux et le politique pose un certain nombre de problèmes largement débattus dans la littérature sociologique et anthropologique. Les travaux réalisés notamment sur des terrains africains (Cruise O’Brien 1981, 2003 ; Piga 2003 ; Kane et Triaud 1998 ; Bayart 1993) montrent bien la variété des modes d'investissement politique du religieux - mise en sens des rapports de domination, modes d'opposition au pouvoir d'État, élaboration de nouvelles légitimités politiques, réaménagement des rapports sociaux. Dans certains cas, le religieux peut devenir l'idiome privilégié du politique, notamment des pratiques de résistance, ou l'instrument le plus efficace de sa négation.

J'adopterai ici une définition du politique qui peut sembler assez large, mais devrait permettre d'échapper aux apories du «tout est politique » (Bayart 1981). Seront considérées comme relevant du politique l'ensemble des pratiques liées au contrôle des rapports de domination. Les ensembles pratiques (dispositifs) empruntent des formes différentes selon la position des groupes et les ressources matérielles et symboliques dont ils disposent en position dominante, construction et renforcement des appareils de pouvoir et de leur

5. «Fétiche» (en bambara boli), « féticheur» (bolitigi), ces termes sont utilisés localement en milieu urbain, aussi bien par les musulmans que par les adeptes des religions traditionnelles. 
légitimation, en position dominée, aménagement, contournement, résistance, ou tentatives de construction d'une contre-hégémonie. "En bas », ces procédures entretiennent des liens étroits, perçus ou non par les agents, avec " la politique » (les politiques publiques notamment), avec le regard porté sur l'appareil d'État et ses élites, avec aussi le regard porté de l'extérieur sur les dispositifs locaux.

Ainsi, le politique ne sera pas considéré comme un secteur particulier de la vie sociale, mais comme "un ensemble de principes générateurs des relations que les hommes entretiennent entre eux et avec le monde » (Lefort 2001 : 8). Reprenant les réflexions platoniciennes sur la notion de politeia, C. Lefort désignera par le terme de "régime ", l'articulation particulière d'une structure légitime de pouvoir et d'un " style d'existence », mode de vie orienté par « les mœurs et croyances qui témoignent d'un ensemble de normes implicites commandant la notion de ce qui est juste et injuste, bien et mal, désirable et indésirable, noble et bas " (Lefort 2001 : 8). Dans cette perspective, on voit bien que la définition de ces normes représente un enjeu fondamental et doit susciter des conflits qui traversent l'ensemble d'une configuration sociale. L'institution de la société, processus de mise en sens et de mise en scène, constitue le politique en phénomène diffus, que l'on ne saurait assigner à un lieu particulier du social ni attribuer à une catégorie spécifique d'agents sociaux.

Selon ces quelques éléments de définition, les dynamiques religieuses dont il sera question ici relèvent aussi du politique. Cette dimension n'échappe pas plus aux fidèles concernés qu'à ceux qui les observent, au sein du quartier comme à l'extérieur. Il s'agit bien dans tous les cas de mettre en œuvre et de diffuser les normes qui doivent orienter la mise en ordre de l'ici-bas et les rapports entre les êtres.

Toutefois chacune des pratiques relevant explicitement du religieux - marquage et occupation de l'espace urbain, réaménagement des rapports sociaux intimes ou publics, du rapport au travail, des conceptions de l'autorité - peut être comparée à un palindrome donnant à voir des significations différentes selon le sens de lecture. Les fidèles, selon les situations observées, selon les contextes de recueil des discours par le chercheur, privilégient tantôt le sens religieux, tantôt le sens politique qu'elle construit. Tantôt le souci du salut dans l'au-delà, tantôt la conquête de l'existence sociale dans le monde. Reste à éclaircir ce que chaque sens doit à l'autre.

Le quartier de Dianguinabougou est l'un des plus récemment urbanisés de Bankoni, à la limite nord du district urbain de Bamako. Les observations utilisées ici concernent ce quartier qui peut être défini comme une « aire morale ». Vu du centre-ville, il est considéré comme « dangereux », repaire de voleurs où la police refuse de s'aventurer. Pour les habitants au contraire, le quartier a longtemps représenté un espace régi par l'ordre villageois, opposé aux perturbations caractérisant le centre-ville.

Les opérations de lotissement, enclenchées en 1992 dans cette zone d'habitat dit " spontané », y ont suscité des réactions particulièrement violentes ${ }^{6}$.

À Dianguinabougou, comme dans toutes les périphéries urbaines bamakoises, le lotissement a remis en cause les règles coutumières qui fondaient les droits d'occupation du

6. En 1994 et 1995 une partie des habitants ont tenté de s'opposer à l'expulsion d'occupants de parcelles réquisitionnées par la municipalité et ont affronté victorieusement les forces de police avant l'intervention de la brigade territoriale. 
sol $^{7}$. Entre 1970 et 1980, les chefs de terre des villages englobés dans le district urbain ont accordé des parcelles aux citadins chassés du centre-ville par l'augmentation des loyers et aux migrants venus des zones rurales. Les relations d'alliance et de subordination liant les " étrangers » accueillis aux autorités coutumières garantissaient à la fois le droit d'usage des nouveaux habitants et leur statut social, l'autorité des chefs de terre et l'autonomie du territoire villageois.

Une dizaine d'années après, la tension du marché foncier au centre-ville a conduit l'État à exercer son droit prééminent sur le sol $^{8}$. Il s'agissait d'aménager les zones périphériques (tracé de voies notamment) et surtout d'y instaurer un marché foncier " formel » (VieillardBaron 1989 ; Bertrand 1997). Les habitants dont les habitations n'ont pas été détruites par les tracés de voies ont été mis en demeure de payer une « lettre d'attribution » aux autorités municipales afin de légaliser leur droit d'usage de la parcelle, ou de l'acheter en toute propriété. Ces procédures ont provoqué, par vagues successives, la fuite d'une partie des habitants (« déguerpis ») et l'extension des « occupations illégales » de terrains réquisitionnés et déjà mis sur le marché par les autorités municipales tandis que les autorités coutumières se hâtaient de les vendre à bas prix. Cet affrontement de la coutume et de la loi donne aujourd'hui encore une forme particulière aux mobilisations foncières. Les revendications lient le droit à l'occupation du sol, le respect des autorités coutumières, des traditions et de l'autonomie locale, que l'État devrait garantir en contrôlant les stratégies des pouvoirs municipaux (Bourdarias 2007).

L'analyse des récits d'installation recueillis depuis 1993 permet d'appréhender la diversité des trajectoires qui se croisent dans l'espace du quartier et les transformations de la population qui y réside.

Les premiers occupants (avant 1970) sont des cultivateurs, attirés par le marché urbain, qui sollicitent la concession de champs auprès des chefs de terre du village rural de Nafadji. Le " premier occupant ", Dianguina, devient chef de quartier et concède à son tour des terrains. D'abord, dit-on, contre quelques kolas qui fondent les liens d'allégeance coutumiers, puis contre des sommes de plus en plus élevées.

À partir des années 1980, de nombreux chefs de famille, fuyant le centre-ville loti, obtiennent des parcelles. La construction d'un logement leur permet de mettre fin à une période de circulation dans l'espace urbain, de location en location. Ils affirment ainsi leur autorité familiale - ils inaugurent un rapport de transmission - et leur statut au sein de leur lignage. Ils ont alors acquis une position économique qu'ils pensent stable, ouvriers d'une usine d'État, artisans... Pour d'autres, l'arrivée dans le quartier n'est qu'une étape dans un parcours d'errance urbaine. Travailleurs précaires, locataires perpétuellement expulsés, ils fuient la hausse des loyers dans les zones loties et aussi parfois la disparition de leur clientèle, c'est le cas des petits artisans réparateurs, des porteurs d'eau... Une décennie plus tard, tous, sous des formes diverses, ont connu une dégradation de leur situation et la mise en œuvre du lotissement remet en cause la sécurité conquise par les occupants de parcelles. Certains sont au chômage, leurs enfants adultes accumulent les emplois précaires. Activités et relations

7. Sous des formes diverses, des phénomènes semblables ont été observés dans la plupart des villes d'Afrique de l'Ouest.

8. L'État malien se réfère au décret du 15 novembre 1935 promulgué par le pouvoir colonial. Il s'accorde ainsi un « droit latent ou éminent» sur toute terre ne relevant pas du droit écrit. 
sociales tendent à se concentrer dans l'espace du quartier ou des zones « spontanées » les plus proches. L'élaboration d'une tradition villageoise semble alors constituer un outil privilégié de négociation et d'interprétation des tensions sociales. Le village, espace de référence, est défini par l'ordre, la reproduction stable des rapports entre générations, la transparence des liens d'alliance et de subordination. Le quartier de Dianguinabougou est constitué en village lorsqu'il s'agit de définir la ville comme un espace d'insécurité, où rien ne vient modérer le pouvoir de l'argent, celui de l'administration. Les luttes foncières qui se déroulent alors révèlent de multiples formes d'investissement du modèle villageois, elles affirment le primat des règles coutumières d'attribution des terres et des liens sociaux qui en dérivent. Lorsqu'il s'agit de donner une cohérence aux perturbations enclenchées par le lotissement, l'État, la municipalité et leurs agents sont définis comme les incarnations d'un pouvoir extérieur et opaque, on n'en peut appréhender les règles, donc en modérer l'appétit, ou en attendre la moindre redistribution.

Pourtant le quartier appartient bien à la ville quand les habitants le confrontent à l'espace imaginaire du village pour décrire la violence qui s'y installe, la dégradation des rapports entre aînés et cadets, entre hommes et femmes.

À partir de 1995, le déplacement des habitants les plus précarisés favorise l'implantation dans le quartier d'une troisième catégorie d'occupants. Leur parcours urbain apparaît comme un parcours d'accumulation de ressources monétaires, de relations, parfois de titres scolaires. Commerçants, employés d'administrations, techniciens, ils instaurent dans le quartier des relations d'échange hiérarchisées, jouent le rôle d'intermédiaires avec l'administration, les employeurs du centre. Ils étendent en quelque sorte les relations de clientélisme qu'ils ont eux-mêmes nouées dans la ville, et alors qu'ils y occupent des positions relativement dominées, ils se voient peu à peu reconnaître localement un statut de « notable». Les stratégies électorales des partis les constituent en intermédiaires privilégiés et leur permettent d'accéder au statut d'élu municipal. La gestion et la répartition des terres sont conçues comme une composante essentielle de ces responsabilités. Les détournements de parcelles loties par les élus municipaux sont aujourd'hui dénoncés par les habitants les plus démunis et contribuent à argumenter le refus « de la politique et des politiciens ».

En 1998, le lotissement est achevé. Les luttes foncières ont échoué. Les dernières entreprises d'État privatisées ont fait faillite. Les luttes menées par les ouvriers « compressés » pour l'obtention de leurs indemnités n'ont le plus souvent remporté aucun succès.

Les quartiers «spontanés » se caractérisaient par l'homogénéité relative des conditions. Le lotissement se traduit par une hétérogénéité sociale croissante. La transition a été brutale. Le modèle du « quartier-village » protecteur devient inopérant, désormais objet de propos nostalgiques et désenchantés. La présence des nouveaux notables, dont les maisons massives tranchent sur les constructions de banco voisines, rend visible aux yeux de tous l'importance des réseaux de clientélisme extérieurs qui conditionnent la réussite sociale. Cette visibilité peut susciter le refus, la révolte des jeunes adultes. Cependant, on voit s'élaborer de nouvelles normes d'évaluation de la valeur sociale des individus et des familles.

Dans un tel contexte, l'espace familier lui-même, le foyer, le voisinage, sont perçus comme des lieux d'incertitude et de conflit. Les jeunes hommes sont confrontés aux accusations de leurs aînés et des femmes qui les rendent responsables de leur situation de chômeur, de l'échec de leurs projets matrimoniaux, de la propagation de la « nouvelle maladie » (sida). Les jeunes femmes sont soupçonnées par les hommes de se livrer à la débauche, de refuser 
le mariage, de propager la « maladie étrangère », les cadets flétrissent les aînés incapables de jouer leur rôle traditionnel en leur procurant des épouses.

Les individus doivent remodeler la hiérarchisation des espaces de vie et de relations, redéfinir les conditions de l'équilibre, notamment en tentant de construire collectivement de nouveaux cadres sociaux de l'expérience. Différentes traditions religieuses peuvent alors être mobilisées, réaménagées et parfois articulées avec des outils symboliques empruntés, notamment certains éléments de l'imaginaire politique diffusé par les ONG occidentales et les institutions internationales ${ }^{9}$.

Les quelques éléments d'analyse présentés ici s'appuient pour l'essentiel sur des observations réalisées dans la longue durée au sein de l'espace du quartier. Des recherches précédentes m'avaient déjà permis de reconstituer les trajectoires familiales et d'analyser les pratiques économiques de jeunes hommes et femmes qui, au cours des années, se sont intégrés aux différents mouvements religieux implantés dans la zone, circulant parfois rapidement de l'un à l'autre. En participant régulièrement aux réunions de nombreux grins ${ }^{10}$, j'avais également pu accumuler des matériaux concernant leurs conceptions du travail, du politique, des relations matrimoniales et familiales. Dans ces groupes sont fréquemment exposés et débattus (parfois résolus) les conflits familiaux, les difficultés économiques, les problèmes de voisinage vécus par les participants. Dans les années 2000, les pratiques religieuses, les activités des différents mouvements ont constitué un objet de débat privilégié, marquant parfois des ruptures au sein des groupes.

J'ai alors recueilli (2003-2004) des entretiens individuels centrés sur la « conversion », les pratiques sociales et religieuses des individus affiliés. Des observations (2005) ont été menées au siège des différents mouvements religieux (siège de l'Ançar-Dine à Dianguinabougou, zawiya $^{11}$ de Soufi Bilal à Sénou et à Djikoroni Para, tòn ${ }^{12}$ de Soufi Adama à Soutadounou, concession du bolitigi Daouda Yattara à Sébénikoro). Je me suis particulièrement intéressée aux activités quotidiennes des élèves vivant en communauté autour du maître, à la réception des fidèles. Les dirigeants religieux m'ont alors accordé plusieurs entrevues.

\section{L'Ançar-Dine : la mise en ordre de la cité}

En 1991, Cheikh Cherif Ousmane Madani Haidara implante le siège de son mouvement, l'Ançar-Dine, à Dianguinabougou. Au départ simple concession périurbaine, l'établissement s'étend en même temps que se développe l'influence du prêcheur. Les affiliations se multiplient dans le quartier (chrétiens, « animistes »), de nombreux musulmans désertent les mosquées locales, et « la maison de Haidara » devient un pôle d'attraction fréquenté par de nombreux fidèles venus d'autres quartiers périphériques, du centre-ville, puis des villages les plus lointains. Aujourd'hui, de hauts bâtiments « en dur » surplombent les simples constructions qui les entourent.

9. Citoyenneté, société civile, cosmopolitisme...

10. Groupes amicaux fondés sur l'appartenance à une même classe d'âge et de sexe.

11. Etablissement local d'un collectif soufi. Certains auteurs traduiront ce terme arabe par « loge » (Cruise O’Brien 1981 : 12). Ce terme est utilisé par les soufis lettrés.

12. En bambara tòn désigne une association, un groupe organisé autour d'un intérêt commun, il désigne aussi les règles qui régissent le groupe. 
Lorsque l'on analyse les récits du Cheikh et de ses proches disciples, cet investissement progressif de l'espace urbain, puis villageois évoque l'épopée de Muhammad ${ }^{13}$ après l'Hidjra vers Médine, lorsque le prophète construit l'Umma avec l'appui des Muhādjirūn (émigrants) Mekkois et des Ansār (auxiliaires) Médinois. Là aussi, la conquête religieuse ne va pas sans conflits, notamment avec les wahhabites ${ }^{14}$, avec les dirigeants des mouvements islamiques rassemblés au sein de l'AMUPI ${ }^{15}$.

La trame biographique élaborée par le $\mathrm{Cheikh}^{16}$ et diffusée par ses élèves met l'accent sur les voyages lointains au cours desquels le prêcheur diffuse la «vraie foi » et les comportements du "vrai croyant ", sur les obstacles que lui opposent à son retour au Mali des chefs religieux « hypocrites » et un " pouvoir corrompu ». Haidara quitte Ségou et la medersa réformiste de Saad Oumar Toure en 1980, parcourt la Côte d'Ivoire. En 1986, de retour au Mali, il s'installe à Bankoni «parmi les pauvres ». Les dirigeants de l'AMUPI, soutenus par le gouvernement de Moussa Traoré lui interdisent tout accès aux media publics. Il prêche alors dans les rues de Bamako et les villages, commence à enregistrer ses prêches et à les diffuser sur cassettes. À la chute du régime en 1991, il fonde l'association AnçarDine.

Les prêches ${ }^{17}$, en langue bambara, adoptent le ton de la causerie familière émaillée parfois de plaisanteries. Ils invitent les fidèles à une remise en ordre de leur vie quotidienne. En premier lieu, c'est la sphère la plus intime, la plus banale en apparence qui est concernée : les rapports entre les conjoints, la régulation des conflits entre les co-épouses, les rapports d'autorité au sein de la famille, la répartition des ressources. Puis les comportements dans l'espace de proximité doivent rendre visible aux yeux de tous cette réforme de la famille : tenue vestimentaire et attitudes des femmes et des enfants, déplacements dans l'espace urbain et rythmes de vie, rapports au travail et à l'argent ${ }^{18}$ des hommes adultes, relations de voisinage. Le respect des règles religieuses (prières, jeûne, aumône) est évalué à l'aune de cette maîtrise de la sphère domestique.

13. Schème courant semble-t-il dans les récits de fondation de nouveaux mouvements religieux réformistes et traditionalistes.

14. La Wahhabyya (sunnite) est un mouvement influent au Mali, surtout dans les villes. Les fidèles sont cependant peu présents dans la zone urbaine concernée. Les Wahhabites sont considérés comme issus d'une classe de commerçants aisés et d'une élite d'intellectuels arabisants (Amselle 1985 ; Brenner 1993).

15. Association Malienne pour l'Unité et le Progrès de l'Islam. Fondée en 1980, l'association devait englober l'ensemble des mouvements islamiques et permettre la régulation des rapports entre les sphères politique et religieuse. Cheikh Haidara participe aujourd'hui au bureau de cette association.

16. Compte tenu de l'approche adoptée ici, privilégiant les élaborations pratiques et symboliques des fidèles, je ne me suis pas attachée à reconstituer la biographie des leaders religieux, travail qui par contre s'avère pertinent lorsque l'on aborde le phénomène religieux par le biais des stratégies mises en œuvre par les « entrepreneurs religieux ». On trouvera une analyse de la trajectoire biographique de Haidara dans les travaux de Dorothéa Schulz (Schulz 2003).

17. Les quelques éléments présentés ici sont issus de l'analyse de prêches diffusés sur cassettes (1996-2005).

18. L'Ançar-Dine impose entre ses membres la suppression du furu wari (compensation matrimoniale), obstacle au mariage des plus pauvres - au moment de l'enquête la somme exigée par la famille de la fiancée pouvait atteindre 150.000 Francs CFA, tandis que les ressources monétaires des jeunes gens dépassaient rarement 20.000 FCFA par mois. La construction de relations économiques privilégiées au sein de l'association doit donner aux jeunes « l'occasion de montrer ce qu'ils savent faire et de réussir s'ils le méritent ». 
Il s'agit là d'une reconquête, d'une reconstruction de la hiérarchie familiale, qui doit permettre aux hommes et aux femmes, aux aînés et aux cadets de retrouver le statut dont les perturbations sociales les ont dépossédés. Cette remise en ordre peut nécessiter le conflit, la rupture : les anciens doivent être respectés, mais s'ils s'opposent, il convient de s'en éloigner ${ }^{19}$.

Dans les prêches, la référence à la tradition religieuse permet d'interpréter ces perturbations, d'en désigner les causes.

En premier lieu Haidara affirme aux jeunes gens qui l'écoutent qu'ils ne sont responsables en rien des échecs qu'ils subissent, il ne cesse de souligner l'injustice des accusations proférées, qu'il s'agisse du chômage, du célibat prolongé, du recours à la prostitution. Les responsables désignés sont les mauvais chefs de famille, indignes ou ignorants, les mauvais riches qui accaparent les ressources au lieu de les investir, les mauvais gouvernants et audelà, le FMI et les organisations internationales qui imposent leurs contraintes.

Les règles qui régissent le groupe des vrais croyants anticipent l'avènement d'une société à la fois juste et pieuse. L'organisation sociale globale est ainsi conçue comme une extension de la sphère domestique. La mise en ordre du territoire social et religieux affirme le rôle fondamental du regard en instaurant en premier lieu la transparence au sein du groupe luimême, en invitant les fidèles à exhiber aux yeux de tous leur nouveau mode d'existence.

En même temps il semble que ce primat de la transparence permette d'inverser sous un angle particulier les rapports entre gouvernants et gouvernés. L'appareil d'État, l'ordre économique ne sont jamais dénoncés dans les prêches. La frontière passe entre les bons et les mauvais riches, entre les bons et les mauvais responsables politiques, les bons et les mauvais imams. Mais les règles de vie des fidèles leur permettent le jugement et l'évaluation du comportement visible des puissants, de retourner en quelque sorte les accusations dont ils ont été la cible.

Il arrive de plus en plus fréquemment que soit mentionné le terme « citoyen » (en français). Ce terme prend un contenu bien particulier tout en évoquant implicitement le modèle démocratique, affirmé par ailleurs par les instances gouvernementales. Le « citoyen » semble ici défini comme celui qui donne à voir sa capacité à maîtriser les rapports sociaux ${ }^{20}$ et à les conformer à la tradition religieuse - ce qui passe par l'abolition de cette frontière entre espace privé et espace public dont profitent les " hypocrites » pour satisfaire secrètement leurs vices. Il se caractérise aussi par sa capacité à diffuser, au-delà du cercle des croyants, des règles de vies conformes à «l'intérêt public $»^{21}$.

19. De nombreux jeunes gens, mariés ou célibataires, quittent la concession familiale, certains choisissent une épouse parmi les "vrais croyants », sans même en informer leurs parents. Des liens amicaux sont rompus lorsque l'ami refuse de s'affilier au tòn ... Là encore, rappel de l'épopée de Muhammad et de ses compagnons.

20. On peut remarquer que le modèle de l'individu qui s'élabore ici entretient des affinités avec la conception privilégiée par de nombreuses sociétés lignagères en Afrique de l'Ouest. Le statut d'individu remarquable est lié notamment à l'accumulation et à la maîtrise des relations sociales, dans les domaines les plus valorisés par la collectivité (CNRS (éd.) 1973).

21. Ainsi, depuis 2005, le « tapage nocturne » (notion absente jusque-là des discours des habitants) a disparu dans le voisinage du siège de l'Ançar-Dine. Plus de veillées de baptême joyeuses et bruyantes, lors des mariages les fêtes organisées par les femmes cessent au coucher du soleil. Il s'agit, me dira-t-on, de respecter les voisins, d'éviter les dépenses excessives tout en bannissant les comportements indécents. Ceux qui passeraient outre, quelle que soit leur appartenance religieuse, risqueraient d'encourir le blâme du voisinage. 
Pour les fidèles dont j'ai recueilli les récits de trajectoire et de conversion, ce modèle s'oppose à la figure de «l'homme perdu », individu coupé de tout lien donc privé de moyens d'existence - l'homme « transparent», diront certains, celui « que personne ne voit ».

Les récits de trajectoire des convertis sont formulés en termes de ruptures successives, d'échecs. L'inscription territoriale proposée par le collectif religieux constitue une protection. Tout se passe comme si la visibilité instaurée dans le groupe permettait de tenir à distance et de rendre intelligibles les pouvoirs arbitraires responsables des échecs, tout en permettant la conquête de l'existence sociale. Pour les hommes, l'affiliation suscite aussi l'espoir de s'insérer dans de nouveaux réseaux économiques dépassant l'espace restreint du quartier. Les jeunes filles mettront fréquemment l'accent sur l'assurance de « gagner un mari » dont le bon comportement sera garanti par la collectivité. Elles espèrent aussi « être considérées », être protégées des dangers qui dérivent de la polygamie, puisque cette situation représente pour elles une catastrophe prévisible et redoutée. Les prêches, en effet, incitent les maris à traiter les co-épouses et leurs enfants avec équité.

Dans ce cadre, la conquête du salut dans l'au-delà semble se confondre avec la conquête du statut dans le monde - à la fois signe d'élection et source d'émotion religieuse.

Toutefois les vertus de la transparence peuvent aisément se retourner contre les fidèles et leur Cheikh. Les non-affiliés, les adversaires, observent et commentent à leur tour les comportements des croyants. Ils détectent avec joie les moindres ambiguïtés, se gaussent des mésaventures (infidélités conjugales, faillites économiques, transgression des normes morales) qui témoignent d'un échec à la fois social et religieux. Les Ançar sont alors soupçonnés de poursuivre les objectifs les plus matérialistes, le Cheikh de dissimuler ses ambitions politiques et économiques. Les défaillances individuelles sont souvent source d'exclusion au sein du tòn. Elles peuvent aussi inciter les membres à rompre avec le groupe et parfois à fuir le quartier.

Les prêches prennent fréquemment pour cible les mauvais imams, et bien sûr, et de plus en plus, les kafiri ${ }^{22}$ et les « féticheurs $»^{23}$. Les soufis ne sont jamais ouvertement évoqués. Les fidèles quant à eux, critiquent avec virulence les comportements vestimentaires des soufis, leur refus de la « vie normale » et de ses devoirs ${ }^{24}$, leur désinvolture vis-à-vis des pratiques religieuses formelles.

Modes d'occupation de l'espace urbain, organisation du collectif, conceptions de la foi, tout en effet semble opposer l'Ançar-Dine et les mouvements soufis qui s'implantent aujourd'hui dans le quartier et proposent un autre modèle de mise en ordre du monde.

22. Terme englobant ici les « animistes » (« païens ») et les « sans religion ».

23. Depuis 2003 environ, les récits de conversions de féticheurs endurcis opérées par Haidara se multiplient, les événements les plus marquants sont filmés et diffusés sur cassettes et DVD.

24. Ces critiques s'adressent aux soufis vivant en communauté, regroupés autour de leur karamogo. « Ils sont là à chanter toute la journée et à manger ce qu'on leur donne ! Ce sont des paresseux !» (membre de l'Ançar-Dine, chômeur, 30 ans, 2005). 


\section{Soufi Bilal et Soufi Adama : la construction d'un réseau d'individus «branchés"}

La métaphore du branchement est aujourd'hui fort prisée des jeunes disciples des deux $k_{a r a m o k o}{ }^{25}$. Elle a été précédée d'images issues de la mécanique automobile ${ }^{26}$. Aujourd'hui, l'exhibition du téléphone portable permet de signifier que les relations qui lient les disciples et leur maître, les disciples entre eux, sont directes et indépendantes des espaces parcourus et des distances, tout comme les relations qui s'instaurent entre l'individu croyant et Dieu. Le groupe des fidèles se libère ainsi des contraintes spatiales.

De nombreux travaux ont souligné le rôle essentiel joué par les confréries soufies dans le processus d'islamisation de l'Afrique sub-saharienne, de même que la multiplicité des traditions auxquelles elles se réfèrent. Certaines, comme la Qadiriyya, étaient déjà implantées au $18^{\mathrm{e}}$ siècle. Mais ces ordres religieux se sont multipliés au $19^{\mathrm{e}}$ siècle. Cruise O'Brien lie leur extension à la constitution d'un espace musulman africain relativement autonome, face à l'hégémonie religieuse arabe, puis à la colonisation occidentale (Cruise O’Brien 1981 ; Cruise O'Brien et Coulon 1988).

Dans les ordres soufis d'Afrique noire se développent différentes formes de religiosité populaire, intégrant parfois des éléments des cultes pré-islamiques, et fondées notamment sur la vénération des Wali (amis de Dieu) vivants ou de leurs ancêtres. Ces personnages articulent certains des traits principaux du renonçant décrits dans les traités de mystique soufie (Popovic et Veinstein 1986). Le Wali a reçu de son maître (ou par sa naissance) une initiation qui le lie à une voie spirituelle (tarîqa) et qui le conduit à " faire son deuil du monde » tout en lui restant lié de multiples manières (Gaboriau 2002). Il est alors doté de pouvoirs surnaturels et devient l'intermédiaire entre Dieu et les hommes. Il exerce des fonctions à la fois cosmiques et politiques.

Les collectifs soufis se multiplient aujourd'hui dans les villes d'Afrique de l'Ouest. Certains ne se rattachent à aucune confrérie. C'est le cas des deux mouvements étudiés ici.

L'un et l'autre sélectionnent des éléments issus d'une tradition soufie globale ${ }^{27}$ qui, déclarait Soufi Bilal « les rassemblent aux soufis du monde entier, passés, présents et à venir ». Quant à Soufi Adama il insiste sur le fait qu'il est « devenu soufi », lorsque ceux qui venaient le visiter dans sa retraite l'ont désigné ainsi : « J'étais là au bord du fleuve à chercher Dieu, à prier tout seul ... Des gens sont venus, ils m'ont appelé « soufi » et m'ont dit qu'ils étaient soufis ... Voilà, c'est tout. $»^{28}$

25. On ne prendra en compte ici que les deux groupes les plus actifs dans le quartier de référence (prosélytisme, installation de disciples).

26. « Notre maître est le chef mécanicien, nous autres, nous sommes les apprentis et on progresse dans le métier ! Les voitures viennent se faire réparer, ce n'est pas un hasard, toi qui es venue, ton moteur doit avoir des ratés ! » (disciple de Soufi Adama, 2001).

27. La non-proclamation d'allégeance à l'une des nombreuses confréries implantées en Afrique de l'Ouest est peut-être transitoire. En tous cas les thématiques du « retour aux origines », du refus des affrontements entre mouvements sont prégnantes. Peut-être sont elles caractéristiques des mouvements émergents.

28. Conversation informelle, 2005. 
Les récits de trajectoire construits par les deux karamoko ${ }^{29}$ sont classiques : retrait du monde, quête mystique, arrivée progressive des disciples et fondation d'un établissement à la lisière de la ville, dans un espace encore vierge d'urbanisation.

Les traits qui différencient les deux autobiographies renvoient bien à la spécificité de chacun des deux collectifs.

Soufi Bilal Diallo, intellectuel diplômé ${ }^{30}$, mentionne une révélation divine, qui l'a conduit à quitter sa ville natale de Ségou pour voyager dans toute l'Afrique sub-saharienne et méditer sur les tombeaux des Wali $^{31}$. Soufi Adama, illettré, lie sa retraite à l'expérience du malheur et de la maladie. Son père, atteint de la lèpre, a quitté le pays dogon avec sa famille à la recherche d'un furakela (guérisseur), puis s'est installé dans un village chrétien proche de Bamako. Adama quitte le village et vit une période de vie érémitique dans la brousse, au bord du Niger.

Parmi les élèves installés dans la zawiya de Bilal, on trouve une forte proportion d'anciens étudiants ou de lycéens ; les personnalités politiques, les cadres de l'armée et de l'administration sont de plus en plus nombreux parmi les fidèles. Les élèves d'Adama sont pour la plupart d'anciens ouvriers, petits artisans ou paysans, et les fidèles se recrutent essentiellement dans les milieux populaires des quartiers périphériques de Bamako et dans les zones rurales proches. Le rapport aux textes religieux situe les deux maîtres à l'opposé l'un de l'autre. Bilal est un lettré qui a acquis une grande connaissance des traditions soufies ; Adama déclare qu'il ne pourra jamais lire le Coran, et que cela importe peu.

Les modalités d'occupation de l'espace opposent les groupes soufis à l'association de Haidara. Le maître et ses disciples les plus proches sont installés aux confins de la ville - Bilal à Sénou ${ }^{32}$, Adama à Soutadounou - dans un habitat sommaire (cases de banco, huttes de branchages), environnés d'animaux familiers, oiseaux apprivoisés, chevaux, et même moutons, qui « ne sont pas là pour être mangés ", précisent volontiers le maître et ses disciples. Puis au sein de l'espace urbanisé seront implantés de petits établissements où résident des disciples moins avancés qui doivent à leur tour attirer des élèves, faire la preuve de leur charisme auprès des populations. L'espace religieux et l'espace de vie quotidienne des habitants des quartiers coexistent mais ne se recouvrent pas. Rendre visite aux soufis pour obtenir une bénédiction, participer aux prières et aux chants, constitue en soi une rupture par rapport aux espaces-temps de la vie quotidienne.

À un autre niveau, la trajectoire spirituelle des disciples accentue ce modèle de retrait du monde.

29. On trouvera la biographie « officielle » de Soufi Bilal sur le site de la Communauté musulmane des Soufis du Mali, dont il est aujourd'hui le dirigeant $<$ soufibilal.org $>$. Ses nombreuses publications y sont également répertoriées. Benjamin Soarès a réalisé une étude approfondie de la trajectoire et des stratégies de Soufi Bilal (Soarès 2007).

30. Son disciple le plus proche m'affirma que le karamoko était diplômé de l'ENA(Ecole Nationale d'Administration malienne), détail que Bilal ne mentionnait pas en 2005.

31. Ce terme désigne les soufis reconnus comme « amis de Dieu » (c'est-à-dire « saints », selon la terminologie couramment utilisée dans la littérature).

32. En 2005, La zawiya actuelle de Djikoroni-Para (quartier de l'Ouest de Bamako situé au bord du fleuve) était en construction. C'est là que résident aujourd'hui Soufi Bilal et une grande partie de ses élèves. Il s'agit d'une vaste construction en dur qui marque bien l'extension et l'enrichissement de la communauté. 
L'attraction exercée par le karamoko, la recherche de la proximité avec Dieu, s'accompagnent d'un processus de rééducation de l'individu qui vient de connaître une seconde naissance. Rompre avec le monde implique une phase de refus, d'agressivité. De nombreux disciples l'exprimaient ainsi : «Le monde devient dangereux, tu vois le diable partout, il faut repousser les autres »; «La vie avec le Maître et les autres élèves t'apprend à être sûr de toi, alors tu fais la paix avec les gens du dehors et tu deviens tolérant ». Soufi Adama, quant à lui, précisait qu'il faut être déjà bien avancé dans la voie pour savoir « que le diable c'est toi et personne d'autre $»$.

Il semble alors que la quête mystique aille de pair avec la construction d'une nouvelle famille, autour d'un père-modèle, où les positions sont hiérarchisées selon les degrés d'acquisition de la sagesse. Dans cette configuration, les regards sont orientés vers le Maître, les disciples les plus avancés et doivent se détourner progressivement du monde environnant. Il appartient aux habitants de ce monde là de s'engager ou non dans la même trajectoire de rupture.

De ce fait, les riches, les gens de pouvoir, ne font l'objet d'aucun jugement. Les seuls qui doivent susciter l'intérêt sont « les voitures cassées qui souhaitent se faire réparer », les puissants en échec notamment. Ainsi en 2005, Moussa Traoré, le dictateur déchu, visite le groupe d'Adama tous les vendredis ${ }^{33}$. Des policiers, officiers de l'armée, fréquentent régulièrement le groupe de Soufi Bilal après avoir compris, déclarent-ils, les méfaits que leurs fonctions les avaient conduits à commettre.

Cette inversion des regards et de la force d'attraction remet en cause les liens de clientélisme qui conditionnent la réussite sociale en milieu urbain. Ainsi se dessine en creux l'évaluation du monde politique et économique, les voies de sa réforme ${ }^{34}$ et le rôle dévolu aux croyants dispersés dans le monde.

Les récits de trajectoire sociale construits par les disciples, comme dans le cas des Ançar, sont scandés d'échecs, d'expériences de l'injustice. Toutefois le thème de la rupture volontaire, antérieure à l'apparition de la vocation religieuse, des liens familiaux apparaît fréquemment. Surtout chez Soufi Adama, un certain nombre de disciples déclarent avoir connu des phases de délinquance. Ils disent tous avoir trouvé là un refuge, une "vraie famille » qui leur permet de dépasser les limites étroites du monde social. Ils dépeignent ce dernier comme violent, sans pitié et voué à la monotonie. L'image du réseau unissant les soufis du monde entier leur donne l'espoir de franchir les frontières, pour devenir, déclarent de jeunes disciples, des « citoyens du monde ».

Avant 2005, Adama et ses disciples affirmaient qu'ils participaient de toutes les traditions religieuses, qui se valent en ce qu'elles incitent les croyants à chercher la même chose. La « religion traditionnelle des anciens bamanan » n'était pas exclue de ce vaste ensemble ${ }^{35}$. Adama a élu domicile sur une colline surplombant un gué traversant le Niger, endroit où les bolitigi effectuent de nombreux sacrifices et où lui-même va méditer toutes les nuits.

33. En 2007, j'ai pu constater qu'il avait établi des liens privilégiés avec Soufi Bilal, dont le prestige ne cesse de croître et dont le mouvement s'est implanté dans plusieurs pays d'Afrique de l'Ouest.

34. Que les hommes et les femmes tournés vers Dieu se multiplient, et le monde changera de lui-même. Les soufis développent volontiers cette thématique.

35. De tels phénomènes de syncrétisme n'ont rien de nouveau ni d'exceptionnel, mais la situation dans laquelle ils se manifestent ici leur donne un sens particulier. 
En 2003, il recevra la visite du « féticheur » le plus honni des dignitaires musulmans, (Daouda Yattara dit Sitane) qui de son côté vantera sur les ondes ${ }^{36}$ sa bonté et sa tolérance.

\section{Sitane (Satan) : l'universalité de la coutume bamanan}

Les chasseurs sont devenus l'un des symboles privilégiés de la «tradition du Mandé ». Dans chaque association aujourd'hui, l'initiation des membres implique l'attachement à un maître : acquisition des savoirs cynégétiques, mais aussi des savoirs ésotériques permettant d'établir des liens avec les forces qui régissent les processus naturels. En ce sens, chasseurs et bolitigi entretiennent de nombreuses affinités. Les uns et les autres se posent en médiateurs entre le monde des hommes et celui des esprits (Cissé 1964).

Depuis quelques années, les bolitigi se multiplient dans les zones urbaines périphériques. Les mouvements de chasseurs deviennent de plus en plus visibles (Arseniev 2007). Le processus de patrimonialisation de la tradition enclenché par l'État Malien a donné lieu à l'organisation de festivals, de «Fêtes des chasseurs », à l'érection d'un monument à leur gloire dans le centre-ville.

Toutefois, dans les quartiers périphériques, la mobilisation de la tradition (confortée par la politique étatique) est investie de sens bien différents. Et entre autres d'un sens explicitement politique. Un certain nombre d'événements (2003-2006) ont attiré mon attention sur ce phénomène, en particulier des "meetings » de chasseurs organisés dans les quartiers, où étaient prononcés des discours particulièrement agressifs, dénonçant l'oppression exercée par les musulmans liés au pouvoir en place, préconisant le retour à la société paisible et égalitaire « d'avant la conquête musulmane »-le tout ponctué d'horribles blasphèmes qui semblaient réjouir les nombreux assistants. Recours aussi, en 2003, à une société de chasseurs pour protéger un marché qui venait d'être pillé par des bandits ${ }^{37}$.

Si alors de nombreux bolitigi s'exprimaient sur les ondes des radios indépendantes, l'un d'entre eux semblait avoir conquis un prestige particulier - large diffusion de ses cassettes sur les marchés, multiplication du nombre de ses élèves dans les quartiers, attaques répétées de la part des dignitaires religieux dans les prêches et dans la presse. Fait intéressant, Daouda Yattara s'était attribué le nom de Satan (Sitanè), inscription tenant lieu, sur son véhicule, de plaque d'immatriculation.

Le siège de Satan (Sitanébougou) s'élève à la lisière sud de la ville, à Sébénikoro, à côté de la centrale électrique. Avant d'obtenir quelques entretiens, j'ai pu passer là-bas de nombreuses journées qui m’ont permis d'évaluer le nombre impressionnant de ceux qui venaient solliciter ses services, " animistes », musulmans et chrétiens confondus. Dans la cour, entre deux entretiens privés, Satan rendait la justice, faisait châtier ${ }^{38}$ par ses aides les voleurs que les propriétaires lésés conduisaient chez lui.

La biographie présentée par Daouda Yattara est celle d'un « jeune homme en colère » qui dit avoir été confronté dès son enfance à l'injustice et à l'oppression. Les événements

36. Différentes radios privées diffusent alors ses déclarations, notamment radio Kayira et radio Jekafo.

37. Le rôle des milices de chasseurs en Afrique de l'Ouest a fait l'objet d'un certain nombre de publications (Ferme 2001).

38. Quelques coups de fouet appliqués avec une certaine « bienveillance » : «Allez, courage ... encore trois coups et c'est fini ! Tu pourras dormir tranquille après ». 
qui articulent les récits mettent en scène une famille rurale appauvrie et exploitée par de riches musulmans ${ }^{39}$. Si la scène de la révélation, le récit de formation de maître en maître sont des plus conventionnels ${ }^{40}$, les réaménagements de la tradition religieuse opérés ici le sont beaucoup moins.

Les croyances des « vieux bamanan », religion respectueuse de la nature de l'homme - être dont les besoins sont légitimes et doivent être satisfaits - entretiennent une lutte séculaire contre les religions monothéistes ${ }^{41}$, qui « divisent» les hommes et dont la seule fonction est de réprimer cette nature. La référence constante à Satan doit sans doute être interprétée dans ce cadre. Les défaites de la religion traditionnelle viendraient de ce que les bolitigi, qui sollicitaient les forces de la nature au profit de la collectivité villageoise, se les sont appropriées en ville pour leur usage personnel. La restauration de la religion passe donc par la restauration de la coutume villageoise et des liens sociaux qu'elle impliquait.

Si les intérêts sociaux (la conquête des pouvoirs politique et économique individuels) divisent et isolent les hommes, il apparaît dans ce discours que les besoins (mago) ${ }^{42}$ fondent la ressemblance entre tous les êtres et leurs liens avec la nature. En ce sens, me fera-t-on souvent remarquer, la coutume bamanan est valable pour tous, dans le monde entier.

De nombreux bolitigi précisent que les musulmans qui viennent les consulter en secret témoignent de la force de ce que leur religion réprime. Ainsi « la coutume progresse dans l'ombre ».

En avril 2005, Sitanè a été accusé de complicité de meurtre, puis de trafic d'organes humains. Il a été condamné en 2007 à cinq années d'emprisonnement ${ }^{43}$. Cet événement a rapidement été constitué en affaire d'État. D'autant que d'autres arrestations ont été effectuées (officiers de l'armée et de la police notamment). L'analyse des articles de presse ${ }^{44}$, montrant bien les pressions exercées par les hiérarchies religieuses et l'embarras du pouvoir d'État, permet de déceler la dimension politique conférée « en haut » à ce qui a parfois été qualifié de « complot souterrain » contre l'ordre social et la démocratie. Dans les zones périphériques, les causeries entre amis et voisins étaient l'occasion d'affrontements entre les tenants de la défense de l'Islam et ceux qui accusaient le pouvoir étatique de complot contre

39. La famille de Daouda Yattara est originaire de la région de Gao et s'est installée dans le Bélédougou.

40. Daouda dit avoir été témoin de l'humiliation de son père et de sa mère par le propriétaire terrien musulman qui les employait. Durant la nuit qui a suivi cet événement, un esprit lui est apparu. Il entreprendra alors de « rechercher le savoir » auprès des bolitigi du Bélédougou, puis de Guinée.

41. Ici l'ennemi désigné est l'Islam, car « le christianisme, maintenant, laisse les gens tranquilles ».

42. Semblent ainsi être opposés le désir d'imposer sa loi aux autres (politique) et le « besoin ». Interrogés sur ce point, Satan et ses disciples énumèrent : « faire que ton champ ou ton commerce gagne beaucoup, que tu gagnes au tiercé, que tu puisses avoir la femme que tu aimes et qu'elle te soit fidèle, que tu guérisses de ta maladie... »

43. Il a été discrètement libéré en mai 2009. La presse malienne n'a pas mentionné cette libération.

44. On trouvera de nombreux articles publiés par les journaux maliens entre 2005 et 2009, en consultant le site $<$ Maliweb.net $>$. 
les pauvres ${ }^{45}$. Aujourd'hui encore, des groupes de jeunes écoutent, le soir, les cassettes de Sitanè. Pour les imams les plus médiatiques, la conversion du prisonnier était devenue un enjeu.

Ce dernier mouvement est difficilement comparable aux précédents. Il ne suscite pas la formation d'un collectif stable, repérable dans l'espace de la ville et ne propose pas, bien entendu, une voie vers le salut. Il ne se constitue pas en " aménageur » de l'espace urbain et des relations. Les références au « village de la tradition" semblent relever à la fois de la nostalgie et de la convention. La localisation des pratiques cultuelles (espace domestique, demeure des bolitigi locaux), les manifestations publiques des associations de chasseurs, révèlent les représentations d'un milieu urbain fragmenté, hostile, conflictuel ${ }^{46}$. Il ne s'agit pas pour l'heure d'y conquérir un territoire d'appartenance, mais de susciter « dans l'ombre » l'adhésion des individus.

\section{Conclusion}

L'interdépendance conflictuelle des mouvements religieux sélectionnés ici peut être considérée selon différentes échelles d'observation et d'analyse.

Adoptons une « focale moyenne », prenant pour objet la configuration religieuse locale, on voit alors que tous recrutent des fidèles dans les populations les plus précarisées des quartiers périphériques. Tous définissent leur spécificité en sélectionnant et en articulant les traits d'une pratique islamique formelle, désignant ainsi les formes religieuses et sociales qu'ils entendent combattre. Chaque collectif religieux fonde son autorité sur le rattachement à une lignée croyante (Islam, religion des anciens bamanan), propose aux adeptes un retour à un univers de significations collectives immuable. La conquête de la légitimité procède du respect de la tradition religieuse dont le dévoiement serait à la fois le principe et l'effet du désordre social vécu.

Les quelques éléments d'observation présentés plus haut indiquent un processus de sélection et de réinvestissement des éléments pertinents de ces traditions, choisis à un moment donné pour leur "valeur d'usage " lorsqu'il s'agit de donner du sens aux perturbations sociales et aux conflits qui traversent le champ religieux. Ces constructions sont bien entendu

45. D’après mes observations, les adeptes de la « religion bamanan » constituent un groupe très hétérogène : villageois récemment urbanisés ; individus en échec social, souvent « transfuges » provenant de mouvements islamiques; membres des nouvelles couches moyennes occupant des positions plus ou moins stables dans l'échelle sociale. De ce fait la diversité des interprétations et des usages de la tradition mériterait une analyse approfondie.

46. Lors des réunions tenues dans les quartiers périphériques (2005-2007), les sociétés de chasseurs mettaient en scène ponctuellement leur présence et leur puissance, dans un espace local quadrillé par les mouvements religieux et les partis politiques dont ils contestaient la légitimité et les principes. Par les violences verbales, les blasphèmes adressés à la religion dominante, les manifestants affirmaient qu'ils « ne craignent rien ni sur terre ni ailleurs ». Lors des entretiens, bolitigi et adeptes laissent entendre que « ce qui se passe ici (en ville) » ne peut être aujourd'hui réformé. 
relativement instables. Elles sont réajustées au gré des accusations formulées par l'adversaire commun, de l'évaluation des forces et des faiblesses des mouvements concurrents ${ }^{47}$.

Les multiples transferts d'allégeance entre les mouvements permettent quelques réflexions concernant les formes microsociales d'articulation du religieux et du politique. Les individus concernés lient toujours explicitement leur décision aux relations sociales qui prévalent dans chaque tòn religieux, au statut social lié à l'appartenance.

Les femmes qui délaissent l'Ançar-Dine pour rejoindre un groupe soufi déclarent souvent qu'elles ont trouvé là un groupe où « on est moins soupçonneux envers les femmes », où " l'amitié et la gaîté entre hommes et femmes est possible ». Des hommes en échec, dévalorisés au sein de l'Ançar-Dine, prendront le même chemin ou « reviendront à la religion des ancêtres ». Les passages d'un groupe soufi à un autre sont rapportés au prestige social du karamoko, à l'étendue du réseau dont il dispose au Mali et à l'étranger.

Les mouvements - et pour les mouvements islamiques la voie vers le salut qu'ils proposent - sont donc évalués à l'aune de l'existence sociale qu'ils garantissent, de la protection qu'ils assurent contre les contraintes de l'ordre économique et social.

Dans cette situation particulière, il semble que la perception de ces contraintes et de ce qui les génère oriente les constructions religieuses, la sélection et l'appropriation des éléments de tradition.

De tels processus se développent localement au moment où les mouvements de lutte dirigés contre l'État ont été mis en échec (révoltes foncières, militantisme, pour certains, dans les partis politiques, luttes syndicales), où la crise économique étend son emprise.

Les dynamiques religieuses peuvent alors constituer une forme de manifestation du politique. Il s'agit bien de reconfigurer les relations sociales locales, d'élaborer une légitimation de ce nouvel ordre en articulant des principes qui transcendent le local, relevant de l'universel (universalité de la religion monothéiste, universalité de la « religion traditionnelle »). En même temps sont désignées les causes du désordre. Les figures de l’État et des élites qui se dessinent apparaissent en creux à travers les regards qui les évaluent (Ançar-Dine), qui s'en détournent (soufis), à travers les jeux du secret et de l'exhibition propres aux adeptes de la coutume bamanan, à travers aussi les formes d'organisation sociospatiales.

On ne saurait toutefois constituer ces phénomènes en productions sociales et symboliques autonomes. Les mobilisations religieuses entretiennent des liens d'interdépendance étroits avec la sphère étatique et les conflits qui s'y développent.

Ainsi les politiques publiques d'aménagement de l'espace urbain, les orientations économiques mises en œuvre par l'État imposent un cadre contraignant aux dynamiques sociales locales qu'elles contribuent fortement à orienter. Elles proposent aussi une bonne partie des matériaux ${ }^{48}$ dont s'emparent les dispositifs locaux - énoncés politiques, éthiques

47. Les indices de ces ajustements sont multiples et nécessiteraient une description minutieuse, ils font l'objet d'une recherche en cours. Depuis 2005 en effet, les relations que les mouvements musulmans entretiennent entre eux et avec l'État ont connu des transformations notables. Ajoutons à cela qu'à un certain stade de son développement, un mouvement religieux contestataire tente fréquemment d'acquérir une légitimité auprès d'une population plus large et plus diversifiée. C'est aujourd'hui semble-t-il le cas le l'Ançar-Dine et du mouvement de Soufi Bilal.

48. Thématiques de la pacification des rapports sociaux, de l'aménagement rationnel de l'espace urbain, de la lutte contre la délinquance, de la valorisation des traditions nationales ; notions de bien public, de citoyenneté. 
qui seront tantôt retournés, tantôt réinvestis, articulés à des énoncés inscrits dans d'autres sphères, ici le religieux.

Les mobilisations locales, en retour, conduisent à des réaménagements des politiques publiques, des discours de légitimation étatiques. Lorsqu'elles concernent les enjeux politiques fondamentaux, elles peuvent aussi durcir les conflits qui opposent les différentes fractions des élites. Les relations entre l'État et la sphère religieuse constituent l'un de ces enjeux. Les débats concernant la notion de laïcité, l'autonomie du politique, traversent les formations politiques alliées au sein de l'appareil d'État. La prolifération de mouvements islamiques réformistes et néo-traditionalistes accentue ces tensions.

Il a été choisi ici de privilégier l'analyse des configurations religieuses à l'échelle locale, on voit bien cependant que la compréhension des formes d'articulation du politique et du religieux qui s'y élaborent impliquerait la prise en compte d'espaces d'observation plus larges englobant les associations religieuses nationales et les rapports qu'elles entretiennent avec l'État, et au-delà les réseaux transnationaux en formation.

La question de l'autonomie des formes religieuses et sociales observées n'est donc guère pertinente. Par contre la volonté d'autonomie, les compétences symboliques et sociales des populations concernées se lisent dans les modalités de sélection et de mise en sens des outils proposés par les traditions, par les discours politiques et sociaux extérieurs. Elles sont également perceptibles dans la rapidité des réajustements symboliques opérés, dans les changements d'affiliation des individus et des groupes, dans la multiplicité des formes d'action politique investies.

\section{Références citées}

Amselle, Jean-Loup, 1985. « Le Wahhabisme à Bamako (1945-1985) », Canadian Journal of African Studies, p. 345-357.

Arseniev, Vladimir, 2007. «Les chasseurs Donso du Mali à l'épreuve du temps », Afrique Contemporaine, 3-4, (223), p. 341-361.

BAYART, Jean François,

(éd.), 1993. Religion et modernité politique en Afrique Noire. Paris : Karthala.

1981. « Le politique par le bas en Afrique Noire », Politique Africaine, 1, p. 53-82.

Bertrand, Monique, 1997. « Bamako, entre extensions et rattrapages », Etudes foncières, 76.

Bourdarias, Françoise,

1999. «La ville mange la terre. Désordres fonciers aux confins de Bamako (Mali) », Journal des anthropologues, 77-78, p. 141-160.

2003. « ONG et recomposition des élites », Journal des anthropologues, 94-95, p. 23-52.

2007. « La décentralisation, la coutume et la loi. Les constructions imaginaires d'un conflit à la périphérie de Bamako (Mali) », in Claude Fay et Catherine Quiminal (éds), Pouvoirs et décentralisation en Afrique de l'Ouest, p. 221-238. Paris : IRD.

BRENNER, Louis, 1993. « Constructing Muslim identities in Mali » in Louis Brenner (éd.), Muslim Identity and Social Change in Sub-Saharan Africa, p. 50-78. London : Hurst.

Cissé, Youssouf, 1964. « Note sur les sociétés des chasseurs malinke », Journal de la société des Africanistes, Tome XXXIV, fascicule II, p. 175-226.

CNRS (éd.), 1973. La notion de personne en Afrique noire. Actes du colloque du CNRS, 11-17 octobre 1971. Paris : CNRS/L'Harmattan. 
Cruise O'Brien, B. Donal,

1981. « La filière musulmane. Confréries soufies et politique en Afrique noire », Politique Africaine, 4, p. 7-30.

2003. Symbolic confrontations : Muslim imagining the State in Africa. London : Hurst and Company.

Cruise O’Brien, B. Donal et Christian Coulon (éds), 1988. Charisma and Brotherhood in African Islam. Oxford : Clarendon Press.

Ferme, Marianne, 2001. « La figure du chasseur et les chasseurs-miliciens dans le conflit sierra-léonais », Politique Africaine, 82, p. 27-48.

GABORIAU, Marc, 2002. " Incomparables ou vrais jumeaux ? Les renonçants dans l'hindouisme et dans l'Islam », Annales, 57 (1), p. 71-92.

Kane, Ousmane et Jean Louis Triaud (éds), 1998. Islam et islamismes au sud du Sahara. Paris : Iremam-KarthalaMSH.

Lefort, Claude, 2001. Essais sur le politique. Paris : Seuil, Points Essais.

PigA, Adriana (éd.), 2003. Islam et villes en Afrique au sud du Sahara. Paris : Karthala.

Popovic, Alexandre et Gilles Veinstern, (éds), 1986. Les ordres mystiques dans l'Islam. Cheminements et situation actuelle. Paris : Editions de l'EHESS.

Schulz, Dorothea E.,

2003. « Charisma and Brotherhood » Revisited : Mass-Mediated Forms of Spirituality in Urban Mali », Journal of Religion in Africa, 33 (2), p. 146-171.

2006. «Promises of (Im)mediate Salvation : Islam, Broadcast Media, and The Remaking of Religious Experience in Mali », American Ethnologist, 33 (2), p. 210-229.

SoARÈs, Benjamin, 2007. « Saint and Sufi in Contemporary Mali », in Martin van Bruinessen and Julia Day Howell (éds.), Sufism and the "Modern » in Islam, p. 76-91. London/NewYork : I.B. Tauris.

Vieillard-Baron, Henri, 1989. «Bamako : croissance urbaine et évolution du lotissement », Villes en Parallèle, 14. 